\title{
Antieukaryotic type six secretion system virulence factors of bacteria
}

Silindile Maphosa, Lucy N Moleleki, Thabiso E Motaung

University of Pretoria, Private Bag X20, Hatfield 0028, South Africa

Forestry and Agricultural Biotechnology Institute, University of Pretoria, Hatfield 0083, Pretoria, South Africa.

Correspondence: Email: lucy.moleleki@fabi.up.ac.za; thabiso.motaung@up.ac.za

Silindile Maphosa orcid.org/0000-0002-5072-1530

Lucy N Moleleki orcid.org/0000-0002-1003-4621

Thabiso E Motaung orcid.org/0000-0002-8813-7671

University of Pretoria

Private Bag X20

Hatfield 0028

Pretoria

South Africa 


\begin{abstract}
Gram negative bacteria commonly use the type VI protein secretion system (T6SS) to compete for resources and space. Upon activation, in a contact-dependent manner, toxic virulence factors, generally referred to as effectors, translocate from this secretion system into the competitor prokaryote or eukaryote. While much emphasis has been put on prokaryotic competition mediated by T6SS, very little is known about the effect of bacterial interactions with eukaryotic hosts. Similarly, virulent effectors of T6SS discovered to date are widely considered antibacterial. Understanding how this combination of effectorimmunity pairs affects the recipient eukaryotic organism's physiological processes will provide opportunities to advance T6SS in agriculture and biotherapeutics. Here the biological functions of T6SS virulent effectors are reviewed, with a particular emphasis on bacterial interactions with animals, plants, and fungi. Pipelines that are currently used to characterize antieukaryotic T6SS effectors are further discussed in brief.
\end{abstract}

Keywords: Type VI Secretion System, antieukaryotic effectors, interkingdom competition, virulence 


\section{Introduction}

In different environmental settings, means to optimize fitness, survival, and thrive include multiple common bacterial themes (e.g. rapid growth and biofilm formation), one of the most important being the type six secretion system (T6SS). This multi-protein secretion system is encoded by large and conserved gene clusters in both pathogenic and nonpathogenic Gram-negative bacteria (GNB), ${ }^{1}$ and controls bacterial interaction with microbes, plants, and animals (Fig 1). The T6SS is highly diverse in animal- and plantassociated bacteria, including the GNB of the phylum Proteobacteria. As a result, phylogenetic studies have sub-classified the system into the main Proteobacterial T6SS', Francisella T6SS", and Bacteroidetes T6SS III $^{\text {cluster loci. }}{ }^{2}$

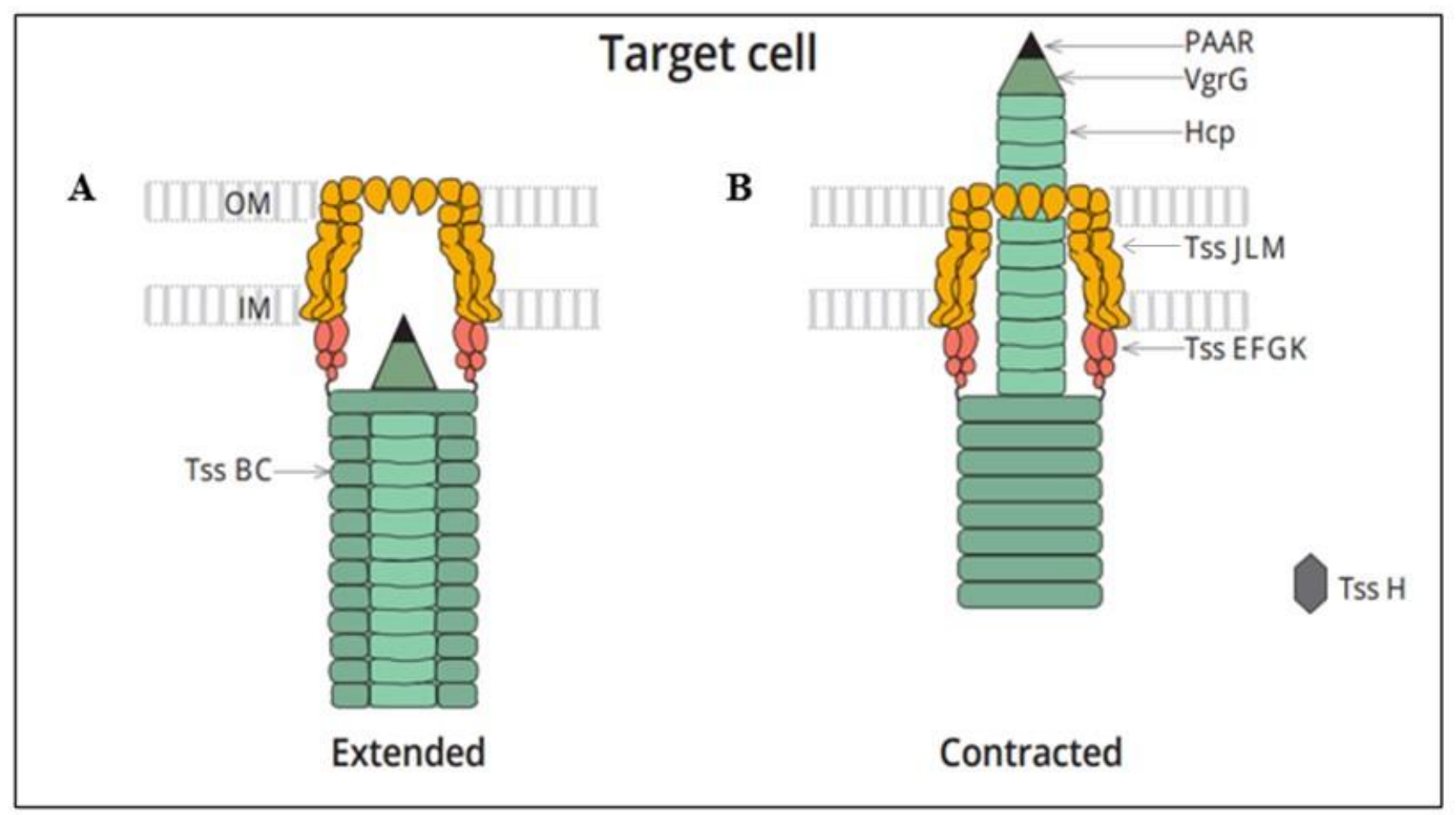

Figure 1: Type six secretion system (T6SS) components TSSA-M. illustrated is the

TSSB/C (tail sheath) that connects to TSSJ/L/M (membrane complex) anchor via TsSEFKG (baseplate) and Tssl (VgrG). The tail sheath surrounds TssD (Hcp tail tube). T6SS in ( $A$ ) extended configuration (B) contracted configuration.

Virulence effectors of the T6SS are surprisingly dependent on "themselves" for secretion, lack $N$-terminal hydrophobic sequences, and sometimes require contact for release. ${ }^{3}$ The 
T6SS and related effectors are critical for bacteria to respond to the environment (Fig 2). This effectively makes the system one of the key regulatory components of bacterial interspecies and interkingdom competition. However, the completion of the T6SS structure, which is incomplete due to a lack of high-resolution structural details, would lead to a detailed understanding of T6SS-mediated host-microbe interactions. ${ }^{4}$ As a result, multiple projects are underway to report on the structures that can provide important steps toward a complete T6SS assembly based on the atomic model. ${ }^{5-7}$

Several reports have shown that a diverse group of antieukaryotic effectors are delivered by the T6SS (Table 1) and facilitate interkingdom communication between species. Such a communication network appears to be important for bacteria, as seen with other secretion systems, to respond to fluctuations in complex environmental conditions. In addition, bacteria, being social species, can use T6SS to continuously make physical contact over space and time with different eukaryotic hosts, resulting in either a mutualistic or parasitic lifestyle. Whether the former or the latter lifestyle prevails over the other can be determined by a range of conditions. But the molecular exchange of proteins during bacterial-eukaryotic interactions (BEIs) will serve as one of the main determinants of antieukaryotic or symbiotic relationships, and this will be supported by increasing discoveries relating to the involvement of secretion system effectors. The essential role of molecular mechanisms governing BEls will likely extend beyond human to plant infecting fungal pathogens to the degree that secretions systems are used to characterize these interactions. ${ }^{8}$

Table 1: T6SS antieukaryotic effectors

\begin{tabular}{l|llllc}
\hline T6SS & Effector & \multicolumn{1}{c}{ Species } & $\begin{array}{l}\text { Organelle } \\
\text { targeted Y/N }\end{array}$ & $\begin{array}{c}\text { Antieukaryotic/Trans- } \\
\text { kingdom }\end{array}$ & Reference \\
\hline & Opi A \& OpiB & Francisella tularensis & $\mathrm{N}$ & Antieukaryotic & 9 \\
& VasX & Vibrio cholera & $\mathrm{Y}$, Membrane & Trans-kingdom & 10 \\
& EvpP & Edwardsiella piscicida & $\mathrm{N}$ & Antieukaryotic & 11,12 \\
& Edwardsiella ictaluri & & & \\
& & & & &
\end{tabular}




\begin{tabular}{|c|c|c|c|c|c|}
\hline H2-T6SS & TplE (Tle4) & Pseudomonas aeruginosa & $\begin{array}{l}\text { Y, Membrane \& } \\
\text { Endoplasmic } \\
\text { reticulum }\end{array}$ & Trans-kingdom & 13 \\
\hline H2-T6SS & PldA (Tle5a) & Pseudomonas aeruginosa & Y, Membrane & Trans-kingdom & 14 \\
\hline \multirow[t]{3}{*}{ H3-T6SS } & PldB (Tle5b) & Pseudomonas aeruginosa & Y, Membrane & Trans-kingdom & 14 \\
\hline & Vpr01580 & Vibrio proteolyticus & Unknown & Antieukaryotic & 15 \\
\hline & Vpr00400 & Vibrio proteolyticus & Unknown & Antieukaryotic & 15 \\
\hline \multirow[t]{15}{*}{ T6SS1 } & Vpr01570 & Vibrio proteolyticus & Y, Actin & Antieukaryotic & 15 \\
\hline & $\mathrm{Tle}^{\mathrm{Vc}}$ & Vibrio cholera & Y, Membrane & Trans-kingdom & 16 \\
\hline & RhsP1 & Pseudomonas aeruginosa & Unknown & Trans-kingdom & 17 \\
\hline & PldB & Pseudomonas aeruginosa & Y, Membrane & Trans-kingdom & 14 \\
\hline & Tle5 $^{\mathrm{KP}}$ & Klebsiella pneumoniae & Y, Membrane & Antieukaryotic & 18 \\
\hline & Pld1 & Klebsiella pneumoniae & Y, membrane & Antieukaryotic & 18 \\
\hline & VgrG1 & Acinetobacter hydrophila & Y, Actin & Antieukaryotic & 19 \\
\hline & TecA & Burkholderia cenocepacia & $\begin{array}{l}\text { Y, Actin } \\
\text { cytoskeleton }\end{array}$ & Antieukaryotic & 20 \\
\hline & TseL & Vibrio cholera & Y, Membrane & Trans-kingdom & 21 \\
\hline & KatN & Enterohemorrhagic E. coli & $\mathrm{N}$ & Antieukaryotic & 22 \\
\hline & VgrG2b & Vibrio cholera & $\begin{array}{l}\text { Y, Membrane, } \\
\text { microtubule } \\
\text { nucleating } \\
\text { complex }\end{array}$ & Trans-kingdom & 23 \\
\hline & VgrG5 & Burkholderia thailandensis & Y, Membrane & Antieukaryotic & 24 \\
\hline & Tfe2 & Serratia marcescens & Y, Membrane & Antieukaryotic & 25 \\
\hline & Tfe1 & Serratia marcescens & $\mathrm{N}$ & Antieukaryotic & 25 \\
\hline & VgrG4 & Klebsiella pneumoniae & $\mathrm{N}$ & Trans-kingdom & 26 \\
\hline
\end{tabular}


Our knowledge of how BEls are facilitated by the transfer of certain compounds such as nutritional metabolites, proteins, and nucleic acids that enable cell-cell communication may be far ahead of our understanding of how secretion systems and bacterially secreted effectors contribute to this complex dialogue. Nonetheless, despite the widespread occurrence of T6SS in GNB and the fact that bacteria live in close association with eukaryotes, evidence supporting the role of T6SS in BEls is not as forthcoming as for other systems. Given the wealth of information on the ecological importance of T6SS, we provide the current state of knowledge on how this system influences the different collaborative scenarios involving bacteria with animals, plants, and fungi.

\section{Current understanding of T6SS effectors and functional diversity}

A secretion system's functional range is characterized by the effector proteins it secretes and the effects afterward. ${ }^{27}$ Likewise, the endless evolutionary arms race between bacteria and their different interacting partners is possibly supported by the variety of T6SS effectors and target responses. Different T6SS gene clusters in different GNB or in the same bacterium encode T6SS, which is assembled into a metaphorical "crossbow" composed of 13 core structural components that are bacterially conserved. ${ }^{28}$ These components include TssA-TssM and Proline-Alanine-Alanine-Arginine (PAAR) motifcontaining proteins that form the membrane-bound sub-assemblies of one or several T6SS complex injection systems (Fig 1). Most of the T6SS cluster encoding bacteria are considered prolific gene exchangers. ${ }^{29}$ In the case of single-copy clusters, the two major T6SS effector genes i.e., hemolysin co-regulated protein (Hcp), a ring-shaped hexamer that recognizes cognate effectors, and valine glycine repeat $G(\mathrm{VgrG})$, used to puncture adjacent cells, are found as duplicates or sometimes as more copies in the genome of GNB. ${ }^{11}$ While there are records of effector redundancy, clusters within the same organism are not capable of completely replacing each other. ${ }^{31}$ Therefore, a lack of cluster redundancy can mean that to attack different targets, one organism must employ different T6SS clusters. This way, the T6SS mediated bacterial interactions may be cooperative, specialized, or competitive. Virulent T6SS effectors can kill both prokaryotic and eukaryotic cells. To this effect, deadly combinations of specific effectors can be loaded directly at different positions or indirectly via adapters to Hcp, docked to VgrG via the 
Proline-Alanine-Alanine-Arginine or PAAR motif-containing module, or as functional Ctermini of evolved Hcp, VgrG or PAAR proteins. ${ }^{4}$

Other implications of functional diversity include contrasting viewpoints as to whether T6SS effectors vary from toxins secreted by this system. The GNB effector proteins are distinguished by their dependence on a secretion mechanism for delivery to eukaryotic targets and an otherwise "subtle" impact on the target cell. In certain situations, this happens within a network of effectors provided by the same system to suppress or manipulate the target for a period of time in a way that is beneficial to the pathogen. For instance, the transport of functionally varied combinations of effectors through separate mechanisms was demonstrated in the Multiple Effector Translocation VgrG (MERV) model. ${ }^{32,33}$ T6SS effectors targeting other bacteria, however, can have lethal effects that stimulate the generation of ROS (reactive oxygen species) in target cells in a way similar to when bacteria are exposed to antibiotics. ${ }^{34}$ Thus, bacterial T6SS effectors may be associated with "brute force" or lethality with respect to this mechanism, causing irreversible effects with high specificity in their biochemical function. Surprisingly, the effects of a bacterial toxin can be observed on living cells by exogenous addition, contrary to the reliance of the effector secretion system. ${ }^{35}$ The T6SSs therefore tend to secrete toxin-like effector proteins with a range of cellular activities, including degradation enzymes, transcription factors, and hormones. However, this is based on contradictory definitions and mechanisms, suggesting the use of both brute force and stealth force by GNB is based on arising needs. ${ }^{36}$

\section{Antianimal T6SS effectors}

In general, the T6SS is viewed as a dedicated antibacterial weapon as reviewed in ${ }^{37}$. Nonetheless, antieukaryotic effectors and those with a dual purpose called trans-kingdom effectors have been discovered in recent years. ${ }^{38}$ The latter is evidenced by effectors, such as Secreted small protein (Ssp3/Tfe1), which can target both prokaryotic and eukaryotic cells (Table 1). In addition, the discovery of the marker for type sIX, or MIX motif, located on the $N$-terminal regions of several virulence effectors secreted by T6SS1 has provided another prime example of T6SS virulence determinants with a putative 
trans-kingdom operation. ${ }^{15}$ Initially, these virulence factors were identified in the marine bacterium Vibrio parahaemolyticus, one of the causative agents of human gastrointestinal disorders. ${ }^{39}$ It seems that MIX effectors are mainly distributed horizontally among marine bacteria, like $V$. proteolyticus ${ }^{40}$ and their discovery was partially motivated by the belief that many T6SSs are not associated with effectors already known. ${ }^{39}$

Trans-kingdom effectors possess phospholipase activity and oftentimes target the cell membrane. These membrane targeting phospholipases form pores that affect cellular integrity (Table1) and include Type VI secretion exported effector $L$ (TseL)/Type six lipase effector 2 (Tle2) and Virulence-associated secretion protein (VasX) (Table 1). The ability of these trans-acting effectors may be due to the presence of ubiquitous target substrates present in both eukaryotic and prokaryotic cell types. To date, due to limited interest in T6SS effectors targeting eukaryotic cells, most known dual effectors are thus membrane targeting. Research may, however, uncover effectors belonging to other functional groups. Because of the T6SS's complexity, flexibility, functional variability, and promiscuity, related effector proteins may prove much more varied than currently recognized. $\mathbf{4 1}$

Presumably, not all T6SS effectors are destructive. Some have subtle effects such as host defenses being exploited and undermined. Antieukaryotic effectors do not fall into distinct groups, unlike antibacterial effectors. Many T6SS effectors belonging to animal pathogens have currently been shown to have the potential to destroy host cells and control their defenses or avoid them. Many studies have shown substantial evidence that T6SS has antieukaryotic activity against mammalian cells. ${ }^{33}$ Manipulation, avoidance, and killing includes interaction between T6SS effectors and eukaryotic cells, as shown in Fig 2. ${ }^{4}$ The eukaryotic targeting T6SS was initially described in Rhizobium leguminosarum, V. cholerae, $P$. aeruginosa, and Edwardsiella tarda, and identified as a virulence factor involved in interactions with eukaryotes. ${ }^{17} P$. aeruginosa effectors PldA and PldB discharged via the $\mathrm{H} 2$ and H3-T6SS respectively are eukaryotic-like phospholipase $\quad \mathrm{D}$ (PLD) that contribute to the invasion phenotype of the pathogen. ${ }^{14}$ Upon release, PldA degrades phosphatidylethanolamine of other bacteria and eukaryotic membranes, making it a dual targeting/trans-kingdom effector. ${ }^{14}$ In other words, this 
effector functions as a toxic component of $P$. aeruginosa, ensuring a competitive advantage and affecting the integrity of host cells during contact with other bacteria. Studies have demonstrated the effects of H2- and H3-T6SS mutations on Caenorhabditis elegans, with both T6SSs leading to $P$. aeruginosa virulence. ${ }^{42}$ Others recorded that PldB had dual targeting characteristics as the effector of $P$. aeruginosa H3-T6SS. ${ }^{14}$ The $P$. aeruginosa VgrG2b effector has been shown to interact with $\gamma$-tubulin, allowing bacterial internalization into epithelial cells. ${ }^{42}$ T6SS-5 from $B$. Thailandensis targets eukaryotes and is important for the melioidosis of murine pneumonia. ${ }^{43}$ The T6SS secreted spike protein VgrG1 C-terminal domain in V. cholerae carries a cross-linked actin C-terminal effector domain that impairs the phagocytic function of host cells. ${ }^{44}$ Taken together, these studies indicate that T6SS has evolved for bacteria to compete with diverse eukaryotic hosts (Fig 2).

\section{Antiplant T6SS effectors}

In phytopathogen-host interactions, the virulent role of T6SS protein effectors remains a hypothesis supported by findings such as an Hcp protein of $A$. tumefaciens DC58, which promotes potato tumorigenesis, and a number of studies showing a decreased phenotype of virulence in T6SS mutant strains, and up-regulation of T6SS cluster genes in wildtype strains during plant host infection. ${ }^{45-47}$ Recent expression profiling data for Pectobacterium brasiliense 1692 show, for example, upregulation of T6SS at different time points during potato tuber infection, which indicates a possible role in pathogenesis. ${ }^{47}$ The T6SS expression has also been documented to be among several regulatory mechanisms controlled by environmental indicators that mimic host conditions. ${ }^{45,48}$ Since their peptidoglycan is too thick to puncture, most T6SSs cannot be used to attack Gram-positive bacteria (GPB). However, it is likely that more GPB adapted effectors have yet to be found. ${ }^{49}$ There is also an issue of whether T6SS effectors have alternative strategies to engage host systems to promote their transport to the cell target substrates based on the size of a plant cell wall. In Xanthomonas oryzea pv. oryzicola GX01, the two T6SS clusters are not required for virulence in rice. ${ }^{50}$ Therefore, there may only be antianimal T6SS cell functions and perhaps the role of the T6SS during plant infection is indirect. ${ }^{\mathbf{5 0} 51}$ Pest invasion and pathogenicity of $P$. protegens $\mathrm{CHAO}$, an 
environmental plant-colonizing bacterium was shown to benefit from T6SS and the two VgrG modules and their respective effectors RhsA and Ghh1. ${ }^{52}$ The conclusion from this study was that the T6SS-dependent changes in the pest insect's gut microbiome allowed the bacterium to colonize the insect and ultimately promote disease. In addition, the research expressed curiosity as to how the bacterium in its original plant host could use such a strategy.

\section{Antifungal T6SS effectors}

Evidence of support for bacterial fungal interactions (BFls) and virulence in other eukaryotic hosts, including plants and animals, is only good for certain secretion systems (e.g., T1SS, T2SS, T3SS, and T4SS), ${ }^{8}$ but appears weak for the T6SS. In certain cases, for the host fungus to survive in the environment, the eukaryotic interaction triggered by bacterial secretion systems may have a supportive role. This is seen in the Burkholderia rhizoxinica endofungal bacterium, where T3SS maintains the relationship between this bacterium and its host fungus, Rhizopus microspores. ${ }^{53}$ Strains of $B$. rhizoxinica that are deficient in T3SS, are unable to reinfect and grow in the host fungus. On the other hand, $R$. microspores strain not colonized by $B$. rhizoxinica due to the T3SS mutation fails to sporulate. However, the ability of $R$. microspores to sporulate is restored when it is infected with a wild type bacterial isolate, signifying the importance of T3SS in the association of the two microbial species. Therefore, during BFIs / BEls, secretion systems may confer fitness benefits, which is something previously thought to be primarily prevalent during interactions only among bacterial species. An increasing body of literature, however, can prompt investigations to look for antieukaryotic T6SS bacterial effectors.

In an attempt to uncover the function of T6SS, maybe a search for antifungal activity in bacteria might benefit from looking at bacteria with antifungal characteristics already identified. One such species is the Gram-negative rod-shaped bacterium S. marcescens. Serratia strains can spread rapidly over the mycelia of zygomycetes in compost heap microenvironments but not ascomycetes, likely as a tendency to interact with Zygomycotan fungi in the environment. ${ }^{54}$ Interestingly, the migration of $S$. marcescens is 
triggered only by contact with the edges of the bacterial colony with mycelia, which means that physical contact is important in this relationship. Of note, both the spread of $S$. marcescens and the killing of the host fungi interacting with this bacterial species are hampered by knocking out the genes that control movement. However, main genes (e.g., Serratia phospholipase PhIA and pore-forming toxin ShIA) working under these regulators are not involved in the actual killing of fungi. ${ }^{54}$ Moreover, secreted $S$. marcescens chitinases, which are highly secreted by this bacterium, have not been found to be involved in mycelia killing. Taken together, these findings point to other processes that may be involved in the killing of fungal cells during the $S$. marcescens-fungi interaction (Fig 2).

T6SS of $S$. marcescens was recently found to be involved in killing the cells of the ubiquitous yeast fungus Saccharomyces cerevisiae, and human fungal pathogens belonging to the genus Candida (i.e., C. albicans and C. glabrata). ${ }^{25}$ This can partially explain, as previously mentioned, the killing of Zygomycota fungi by $S$. marcescens. ${ }^{54}$ Indeed, the strongest evidence that T6SS can exploit BFIs stems from the fact that two T6SS fungicidal effectors, Tfe1 and Tfe2 (Tfe for T6SS antifungal effector) are translocated directly by $S$. marcescens (Table 1 ). Initial evidence concerning the functioning of these antifungal effectors indicates that Tfe1 impairs the metabolism of nutrients and amino acids and causes autophagy, while Tfe 2 impairs the capacity of the membrane but does not introduce any pores in this organelle. ${ }^{29}$ In the background of polymicrobial community studies, further study of Tfe1 and Tfe2 would possibly provide crucial insights into BFIs.

Antifungal virulence factor discoveries have also been made in other bacterial pathogens linked to T6SS factors. These include Hcp, which has been reported to demonstrate antifungal activity against yeasts using in $P$. syringae pv. tomato DC3000 knockout mutants. ${ }^{55}$ For example, it was found that while Hcp appears to be nonessential for DC3000 to colonize and infect host plants, it may be necessary for competition against fungal yeast species, including Cryptococcus members. ${ }^{55}$ It has also recently been established that con-incubation of $K$. pneumoniae Kp52145 with C. albicans and $S$. cerevisiae results in the T6SS-depedent killing of cells from these yeasts. ${ }^{26}$ This study 
showed that VgrG4, and not VgrG2, is a virulence determinant responsible for the antifungal action of T6SS against both yeasts, indicating that vgrG4 could be a frequently used T6SS antifungal factor in K. pneumoniae strains; in quantitative killing assays with C. albicans, vgrG2 or vgrG4 mutant strains did not subvert cell growth, whereas with $S$. cerevisiae, cell growth was not impaired in the presence of vgrG4 mutant growth. ${ }^{26}$ Therefore, we are likely to see unparalleled insights into the function of T6SS antifungal factors and a compendium of target fungi with the support of current resources, including bioinformatics approaches, as discussed below. In the future, these insights will guide the use of these bacterial T6SS factors as antifungals to treat fungal infections in animals and plants. 


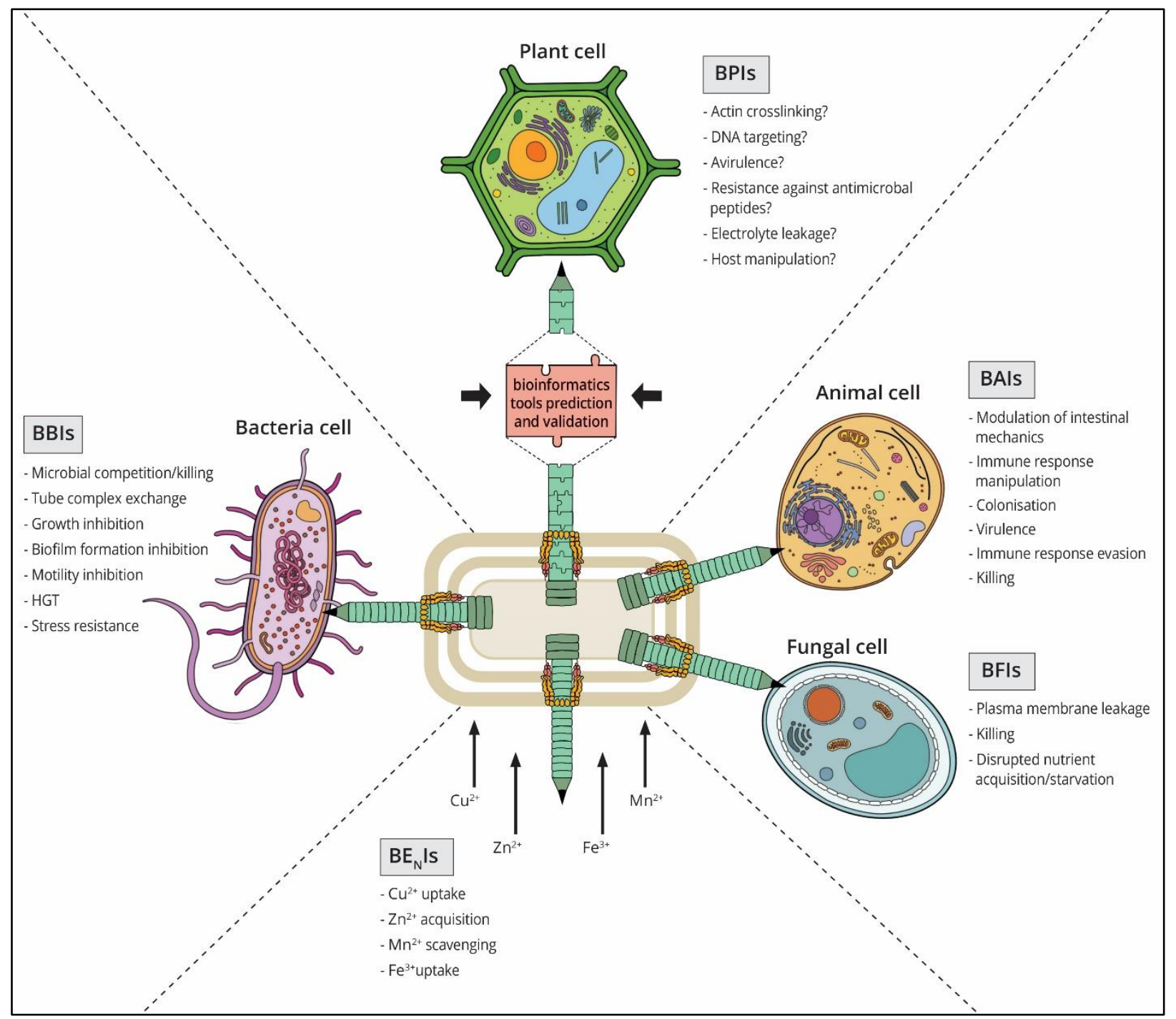

Figure 2: The environmental impact of the type six secretion system (T6SS) entails the regulation of bacterial bacteria interactions (BBIs), bacterial plant interactions (BPIs), bacterial animal interactions (BAIs), and bacterial fungal interactions (BFIs). The T6SS functions also extend beyond these biotic interactions, allowing bacteria to forage key components (e.g. zinc, iron, copper, and manganese) directly from the extracellular environment (BE $I S$ ).

\section{Pipelines currently assisting the discovery of antieukaryotic T6SS effectors}

Information has been provided to understand the distribution and significance of T6SS within species through comparative genomic analyses of T6SS in sequenced bacterial strains. Online bioinformatics databases have been used for the automated annotation of 
T6SS homologues. ${ }^{56}$ Bioinformatics platforms provide a resource for possible effectors using conserved domains/motifs as queries in the databases. ${ }^{57,58}$ The hunt for conserved signaling peptides with bioinformatics tools usually enhances the discovery of putative effectors. Unfortunately, high diversity and the lack of a conserved $\mathrm{N}$-terminal signature sequence presents a challenge in the identification of T6SS effectors. ${ }^{39,59}$ Studies identified $\mathrm{N}$-terminal signal peptide sequences of these effectors, MIX and Found in type sIX effector (FIX). ${ }^{39,59}$ However, validation of MIX reliability was done against Bastion6, the first machine-learning tool that promises accurate prediction of T6SS effectors. ${ }^{60}$ The ability to classify T6SS effectors or significance for translocation and effector function was unfortunately very poor in the MIX motif. ${ }^{60}$ On the other hand, Nguyen et al. used the poorly rated marker and reported 57 novel MIX T6SS effectors and two identified T6SS phospholipases. ${ }^{61}$ Interestingly, in contrast to plant beneficial bacteria, the number of T6SS effectors in Burkholderia plant pathogens was greater and no MIX candidates were found in some of the plant-associated beneficial bacteria. ${ }^{61}$ It is possible that plant beneficial bacteria would have more clusters to provide safety to the plant through outcompeting pathogenic bacteria. T6SS effectors in plant pathogens exhibited high likelihood of association with toxicity on their plant host cells. ${ }^{61}$ Preliminary studies of hostpathogen interactions using the Bastion 6 pipeline (Fig 3) identified 13 T6SS candidate effectors of $P$. parmentieri RNS08.42.1 $\mathrm{a}^{\top}$ potentially targeted at the plant host but not at other bacteria. The prediction and functional characterization of established motifs in silico revealed that the candidate effectors may have potential roles in pathogenic growth and the development of symptoms of disease. For example, it was predicted that one effector will play a role in plant cell RIN4 degradation and consequent activation of RPS2 during bacterial infection. Other effectors have been candidates for promoting plant immune system pathogen evasion, deactivating defense response pathways and mimicking stress signals.

Wang et al. argued that while existing bioinformatics methods are fruitful, they remain restrictive and highly dependent on current knowledge of biochemical properties and transport mechanisms of effectors. ${ }^{60}$ This may be the reason for the lack of confidence, but purported presence of antiplant host targeting T6SS effectors. Simplified statistical approaches are also not appropriate for effector prediction based on sequence similarity, 
patterns, and related gene sequence features. ${ }^{58}$ Universal machine learning methods to reliably predict T6SS effector proteins were proposed as a solution on the basis of these conclusions. Opportunities to further develop bioinformatics methods have been observed since 2018.58,62,63 Three ensemble models were developed by An et al. to evaluate existing tools and enhance their predictive efficiency. ${ }^{56}$ However, these only outperformed tools for the T3SS and T4SS effector predictors and not for T6SS. Zalguizuri et al. made their contribution by validating a phylogenetic profiling method based on the co-evolutionary dependence between the secretomes and the T6SS for effector recognition. ${ }^{62}$ PyPredT6, the python-based prediction tool for T6SS effector protein identification, was developed to overcome the shortcomings of Bastion6. ${ }^{63}$ Wang et al. enhanced the T6SS effector prediction after comparing the efficiency of both Bastion 6 and PyPredT6 by designing a new one. ${ }^{60}$ These authors proposed a more powerful and improved ensemble predictor for identifying T6SS effectors with 100\% specificity, 99\% accuracy, $97.8 \%$ Matthew's correlation coefficient, and $97.1 \%$ sensitivity in independent studies. Therefore, bioinformatics-based approaches to classify new T6SS effectors are a convenient and effective method for the analysis of organisms with sequenced genomes and can facilitate high-throughput detection and functional characterization of candidate effector proteins in collaboration with omics technologies.

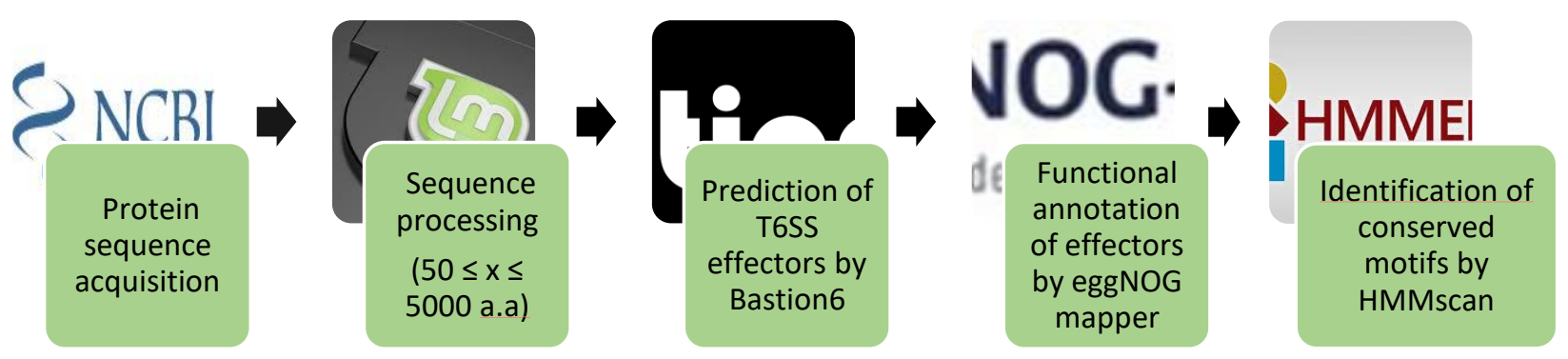

Figure 3: Schematic depiction of antiplant host T6SS effector discovery. Identification of putative effector candidates using Bastion6, eggNOG mapper and HMMscan on the HMMER package for prediction of type six secreted effectors, functional annotation based on orthology and identification of conserved motifs, respectively. 


\section{Conclusions}

As we show in this study, and recently by others ${ }^{64}$ scientific ideology has changed that the T6SS is largely antibacterial. Our challenge, though, is that the T6SS is not only flexible, but it is also complex. There is an accompanying lack of a standard regulatory framework or effector repertoire and function for the system due to its divergent characteristics. This is because, due to the structural, target and mode of action by a variety of effectors, each T6SS assumes a certain uniqueness to the next T6SS. Therefore, in relation to growth inhibition in eukaryotes, we have yet to make captivating discoveries using analytical methods to study T6SS virulence effector proteins. The secreted proteins must be identified and characterized in order to understand the mechanism and the biological significance of any secretion system. ${ }^{27}$ This information will enable us to set goals post research findings and strategies for translation of these outputs.

Pathogens are often studied to monitor their negative impacts or exploit their beneficial functions. Therefore, once T6SS-effectors that target eukaryotic hosts, such as plant crops, are identified, there will be multiple options to explore. The T6SS intersects eukaryotes, prokaryotes and the environment and any addition to this system's knowledge pool can thus contribute to the production of strategies for the management of animal, human, and crop diseases. Resistance genes in distant families of non-hosts may be found in plants with identified effectors or avirulent proteins. These can then be expressed in susceptible targets to confer protection. Protein-protein interaction studies with the help of structural studies can show how the effector and the target interact. Effector-target binding sites may be manipulated so as not to target them without affecting host cell functions. ${ }^{65}$

A great deal has been achieved in understanding the operating principles of the T6SS and its secreted proteins. However, there is still a lot we do not know about the intricate dialogue it mediates between different bacteria, eukaryotic hosts, and the environment. Furthermore, the prediction of putative factors can be enhanced, as seen in the prediction of fungal secretomes, by integrating computer training programs with in vivo expression. ${ }^{66}$ 
Machine learning will in the near future be the key method for the discovery of effectors. Although computational tools cannot substitute experimentation, they can save research funds, time, and resources by providing intel from which informed decisions can be made on a research project. Identified effector candidates can then be easily validated using functional assays.

\section{Funding details}

TEM is supported by grants from the University of Pretoria (Grant No. 1580), DSI-NRF (Grant No. 128877) and Centre of Excellence in Plant Health Biotechnology of Forestry and Agricultural Biotechnology Institute. LNM is supported by DSI-NRF Competitive Funding for Rated Researchers (Grant number 120585).

\section{Acknowledgments}

We are thankful to Glenda Brits for all the graphics used in this paper.

\section{Declaration of interest}

None to declare.

References

1. Cianfanelli FR, Monlezun L, Coulthurst SJ. Aim, Load, Fire: The Type VI Secretion System, a Bacterial Nanoweapon. Trends Microbiol 2016; 24:51-62.

2. Russell AB, Wexler AG, Harding BN, Whitney JC, Bohn AJ, Goo YA, Tran BQ, Barry NA, Zheng H, Peterson SB, et al. A Type VI Secretion-Related Pathway in Bacteroidetes Mediates Interbacterial Antagonism. Cell Host Microbe 2014; 16:227-36.

3. Pukatzki S, Ma AT, Sturtevant D, Krastins B, Sarracino D, Nelson WC, Heidelberg JF, Mekalanos JJ. Identification of a conserved bacterial protein secretion system in Vibrio cholerae using the Dictyostelium host model system. Proc Natl Acad Sci 2006; 103:1528-33.

4. $\quad$ Nguyen VS, Douzi B, Durand E, Roussel A, Cascales E, Cambillau C. Towards a complete structural deciphering of Type VI secretion system. Curr Opin Struct Biol 2018; 49:7784.

5. Ruiz FM, Santillana E, Spínola-Amilibia M, Torreira E, Culebras E, Romero A. Crystal Structure of Hcp from Acinetobacter baumannii: A Component of the Type VI Secretion System. PLOS ONE 2015; 10:e129691.

6. Rapisarda C, Cherrak Y, Kooger R, Schmidt V, Pellarin R, Logger L, Cascales E, Pilhofer M, Durand E, Fronzes R. In situ and high-resolution cryo- EM structure of a bacterial 
type VI secretion system membrane complex. EMBO J [Internet] 2019 [cited 2020 Oct 9]; 38. Available from: https://onlinelibrary.wiley.com/doi/abs/10.15252/embj.2018100886

7. Park Y-J, Lacourse KD, Cambillau C, DiMaio F, Mougous JD, Veesler D. Structure of the type VI secretion system TssK-TssF-TssG baseplate subcomplex revealed by cryo-electron microscopy. Nat Commun 2018; 9:5385.

8. Frey-Klett P, Burlinson P, Deveau A, Barret M, Tarkka M, Sarniguet A. Bacterial-Fungal Interactions: Hyphens between Agricultural, Clinical, Environmental, and Food Microbiologists. Microbiol Mol Biol Rev 2011; 75:583-609.

9. Eshraghi A, Kim J, Walls AC, Ledvina HE, Miller C, Ramsey KM, Whitney JC, Radey MC, Peterson SB, Ruhland BR, et al. Secreted Effectors Encoded Within and Outside of the Francisella Pathogenicity Island Promote Intramacrophage Growth. Cell Host Microbe 2016; 20:573-83.

10. Miyata ST, Unterweger D, Rudko SP, Pukatzki S. Dual Expression Profile of Type VI Secretion System Immunity Genes Protects Pandemic Vibrio cholerae. PLOS Pathog 2013; 9:18. 11. Tan J. EvpP inhibits neutrophils recruitment via Jnk-caspy inflammasome signaling in vivo. $2019 ;: 10$.

12. Kalindamar S, Kordon AO, Abdelhamed H, Tan W, Pinchuk LM, Karsi A. Edwardsiella ictaluri evpP is required for colonisation of channel catfish ovary cells and necrosis in anterior kidney macrophages. Cell Microbiol 2020; 22:e13135.

13. Jiang F, Wang X, Wang B, Chen L, Zhao Z, Waterfield NR, Yang G, Jin Q. The Pseudomonas aeruginosa Type VI Secretion PGAP1-like Effector Induces Host Autophagy by Activating Endoplasmic Reticulum Stress. Cell Rep 2016; 16:1502-9.

14. Jiang F, Waterfield NR, Yang J, Yang G, Jin Q. A Pseudomonas aeruginosa Type VI Secretion Phospholipase D Effector Targets Both Prokaryotic and Eukaryotic Cells. Cell Host Microbe 2014; 15:600-10.

15. Ray A, Schwartz N, de Souza Santos M, Zhang J, Orth K, Salomon D. Type VI secretion system MIX-effectors carry both antibacterial and anti-eukaryotic activities. EMBO Rep 2017; 18:1978-90.

16. Dong TG, Ho BT, Yoder-Himes DR, Mekalanos JJ. Identification of T6SS-dependent effector and immunity proteins by Tn-seq in Vibrio cholerae. Proc Natl Acad Sci 2013; 110:2623-8.

17. Hachani A, Allsopp LP, Oduko Y, Filloux A. The VgrG Proteins Are "à la Carte" Delivery Systems for Bacterial Type VI Effectors. J Biol Chem 2014; 289:17872-84.

18. Lery LM, Frangeul L, Tomas A, Passet V, Almeida AS, Bialek-Davenet S, Barbe V, Bengoechea JA, Sansonetti P, Brisse S, et al. Comparative analysis of Klebsiella pneumoniae genomes identifies a phospholipase D family protein as a novel virulence factor. BMC Biol 2014; $12: 41$.

19. Suarez G, Sierra JC, Erova TE, Sha J, Horneman AJ, Chopra AK. A Type VI Secretion System Effector Protein, VgrG1, from Aeromonas hydrophila That Induces Host Cell Toxicity by ADP Ribosylation of Actin. J Bacteriol 2010; 192:155-68.

20. Aubert DF, Xu H, Yang J, Shi X, Gao W, Li L, Bisaro F, Chen S, Valvano MA, Shao F. A Burkholderia Type VI Effector Deamidates Rho GTPases to Activate the Pyrin Inflammasome and Trigger Inflammation. Cell Host Microbe 2016; 19:664-74.

21. $\quad \mathrm{Fu}$ Y, Waldor MK, Mekalanos JJ. Tn-Seq Analysis of Vibrio cholerae Intestinal Colonization Reveals a Role for T6SS-Mediated Antibacterial Activity in the Host. Cell Host Microbe 2013; 14:652-63. 
22. Wan B, Zhang Q, Ni J, Li S, Wen D, Li J, Xiao H, He P, Ou H, Tao J, et al. Type VI secretion system contributes to Enterohemorrhagic Escherichia coli virulence by secreting catalase against host reactive oxygen species (ROS). PLOS Pathog 2017; 13 :e1006246.

23. Sana TG, Baumann C, Merdes A, Soscia C, Rattei T, Hachani A, Jones C, Bennett KL, Filloux A, Superti-Furga G, et al. Internalization of Pseudomonas aeruginosa Strain PAO1 into Epithelial Cells Is Promoted by Interaction of a T6SS Effector with the Microtubule Network. mBio [Internet] 2015 [cited 2020 Oct 29]; 6. Available from: https://mbio.asm.org/content/6/3/e00712-15

24. Schwarz S, Singh P, Robertson JD, LeRoux M, Skerrett SJ, Goodlett DR, West TE, Mougous JD. VgrG-5 is a Burkholderia type VI secretion system-exported protein required for multinucleated giant cell formation and virulence. Infect Immun 2014; 82:1445-52.

25. Trunk K, Peltier J, Liu Y-C, Dill BD, Walker L, Gow NAR, Stark MJR, Quinn J, Strahl $\mathrm{H}$, Trost M, et al. The type VI secretion system deploys antifungal effectors against microbial competitors. Nat Microbiol 2018; 3:920-31.

26. Storey D, McNally A, Åstrand M, Santos J sa-PG, Rodriguez-Escudero I, Elmore B, Palacios L, Marshall H, Hobley L, Molina M, et al. Klebsiella pneumoniae type VI secretion system-mediated microbial competition is PhoPQ controlled and reactive oxygen species dependent. PLOS Pathog 2020; 16:e1007969.

27. Chen L, Zou Y, She P, Wu Y. Composition, function, and regulation of T6SS in Pseudomonas aeruginosa. Microbiol Res 2015; 172:19-25.

28. Durand E, Nguyen VS, Zoued A, Logger L, Péhau-Arnaudet G, Aschtgen M-S, Spinelli S, Desmyter A, Bardiaux B, Dujeancourt A, et al. Biogenesis and structure of a type VI secretion membrane core complex. Nature 2015; 523:555-60.

29. Thomas CM, Nielsen KM. Mechanisms of, and Barriers to, Horizontal Gene Transfer between Bacteria. Nat Rev Microbiol 2005; 3:711-21.

30. Navarro-Garcia F, Ruiz-Perez F, Cataldi Á, Larzábal M. Type VI Secretion System in Pathogenic Escherichia coli: Structure, Role in Virulence, and Acquisition. Front Microbiol [Internet] 2019 [cited 2020 Oct 10]; 10. Available from:

https://www.frontiersin.org/articles/10.3389/fmicb.2019.01965/full

31. Santos MNM, Cho S-T, Wu C-F, Chang C-J, Kuo C-H, Lai E-M. Redundancy and Specificity of Type VI Secretion vgrG Loci in Antibacterial Activity of Agrobacterium tumefaciens 1D1609 Strain. Front Microbiol [Internet] 2020 [cited 2020 Oct 10]; 10. Available from: https://www.ncbi.nlm.nih.gov/pmc/articles/PMC6971182/

32. Ho BT, Dong TG, Mekalanos JJ. A View to a Kill: The Bacterial Type VI Secretion System. Cell Host Microbe 2014; 15:9-21.

33. Hachani A, Wood TE, Filloux A. Type VI secretion and anti-host effectors. Curr Opin Microbiol 2016; 29:81-93.

34. Dong TG, Dong S, Catalano C, Moore R, Liang X, Mekalanos JJ. Generation of reactive oxygen species by lethal attacks from competing microbes. Proc Natl Acad Sci 2015; 112:21816.

35. Galán JE. Common themes in the design and function of bacterial effectors. Cell Host Microbe 2009; 5:571-9.

36. Jayamani E, Mylonakis E. Effector triggered manipulation of host immune response elicited by different pathotypes of Escherichia coli. Virulence 2014; 5:733-9.

37. Benz J, Meinhart A. Antibacterial effector/immunity systems: it's just the tip of the iceberg. Curr Opin Microbiol 2014; 17:1-10. 
38. Bleves S. Game of Trans-Kingdom Effectors. Trends Microbiol 2016; 24:773-4.

39. Salomon D, Kinch LN, Trudgian DC, Guo X, Klimko JA, Grishin NV, Mirzaei H, Orth K. Marker for type VI secretion system effectors. Proc Natl Acad Sci 2014; 111:9271-6.

40. Salomon D, Klimko JA, Trudgian DC, Kinch LN, Grishin NV, Mirzaei H, Orth K. Type VI Secretion System Toxins Horizontally Shared between Marine Bacteria. PLOS Pathog 2015; 11:e1005128.

41. Silverman JM, Brunet YR, Cascales E, Mougous JD. Structure and Regulation of the Type VI Secretion System. Annu Rev Microbiol 2012; 66:453-72.

42. Sana TG, Hachani A, Bucior I, Soscia C, Garvis S, Termine E, Engel J, Filloux A, Bleves S. The Second Type VI Secretion System of Pseudomonas aeruginosa Strain PAO1 Is Regulated by Quorum Sensing and Fur and Modulates Internalization in Epithelial Cells. J Biol Chem 2012; 287:27095-105.

43. Kollman JM, Merdes A, Mourey L, Agard DA. Microtubule nucleation by $\gamma$-tubulin complexes. Nat Rev Mol Cell Biol 2011; 12:709-21.

44. Ma AT, Mekalanos JJ. In vivo actin cross-linking induced by Vibrio cholerae type VI secretion system is associated with intestinal inflammation. Proc Natl Acad Sci 2010; 107:436570 .

45. Wu H-Y, Chung P-C, Shih H-W, Wen S-R, Lai E-M. Secretome Analysis Uncovers an Hcp-Family Protein Secreted via a Type VI Secretion System in Agrobacterium tumefaciens. J Bacteriol 2008; 190:2841-50.

46. Liu H, Coulthurst SJ, Pritchard L, Hedley PE, Ravensdale M, Humphris S, Burr T, Takle G, Brurberg M-B, Birch PRJ, et al. Quorum Sensing Coordinates Brute Force and Stealth Modes of Infection in the Plant Pathogen Pectobacterium atrosepticum. PLOS Pathog 2008; 4:e1000093.

47. Bellieny-Rabelo D, Tanui CK, Miguel N, Kwenda S, Shyntum DY, Moleleki LN. Transcriptome and Comparative Genomics Analyses Reveal New Functional Insights on Key Determinants of Pathogenesis and Interbacterial Competition in Pectobacterium and Dickeya spp. Appl Environ Microbiol [Internet] 2019 [cited 2020 Oct 10]; 85. Available from: https://aem.asm.org/content/85/2/e02050-18

48. Mattinen L, Nissinen R, Riipi T, Kalkkinen N, Pirhonen M. Host-extract induced changes in the secretome of the plant pathogenic bacterium Pectobacterium atrosepticum . PROTEOMICS 2007; 7:3527-37.

49. Chou S, Bui NK, Russell AB, Lexa KW, Gardiner TE, LeRoux M, Vollmer W, Mougous JD. Structure of a Peptidoglycan Amidase Effector Targeted to Gram-Negative Bacteria by the Type VI Secretion System. Cell Rep 2012; 1:656-64.

50. Zhu P-C, Li Y-M, Yang X, Zou H-F, Zhu X-L, Niu X-N, Xu L-H, Jiang W, Huang S, Tang J-L, et al. Type VI secretion system is not required for virulence on rice but for interbacterial competition in Xanthomonas oryzae pv. oryzicola. Res Microbiol 2020; 171:64-73. 51. Fan J, Ma L, Zhao C, Yan J, Che S, Zhou Z, Wang H, Yang L, Hu B. Transcriptome of Pectobacterium carotovorum subsp. carotovorum PccS1 infected in calla plants in vivo highlights a spatiotemporal expression pattern of genes related to virulence, adaptation, and host response. Mol Plant Pathol 2020; 21:871-91.

52. Vacheron J, Péchy-Tarr M, Brochet S, Heiman CM, Stojiljkovic M, Maurhofer M, Keel C. T6SS contributes to gut microbiome invasion and killing of an herbivorous pest insect by plant-beneficial Pseudomonas protegens. ISME J 2019; 13:1318-29. 
53. Lackner G, Moebius N, Hertweck C. Endofungal bacterium controls its host by an hrp type III secretion system. ISME J 2011; 5:252-61.

54. Hover T, Maya T, Ron S, Sandovsky H, Shadkchan Y, Kijner N, Mitiagin Y, Fichtman B, Harel A, Shanks RMQ, et al. Mechanisms of Bacterial (Serratia marcescens) Attachment to, Migration along, and Killing of Fungal Hyphae. Appl Environ Microbiol 2016; 82:2585-94.

55. Haapalainen M, Mosorin H, Dorati F, Wu R-F, Roine E, Taira S, Nissinen R, Mattinen L, Jackson R, Pirhonen M, et al. Hcp2, a Secreted Protein of the Phytopathogen Pseudomonas syringae pv. Tomato DC3000, Is Required for Fitness for Competition against Bacteria and Yeasts. J Bacteriol 2012; 194:4810-22.

56. An Y, Wang J, Li C, Leier A, Marquez-Lago T, Wilksch J, Zhang Y, Webb GI, Song J, Lithgow T. Comprehensive assessment and performance improvement of effector protein predictors for bacterial secretion systems III, IV and VI. Brief Bioinform 2016; :bbw100. 57. Repizo GD, Espariz M, Seravalle JL, Salcedo SP. Bioinformatic Analysis of the Type VI Secretion System and Its Potential Toxins in the Acinetobacter Genus. Front Microbiol [Internet] 2019 [cited 2020 Oct 11]; 10. Available from: https://www.frontiersin.org/articles/10.3389/fmicb.2019.02519/full

58. An Y, Wang J, Li C, Leier A, Marquez-Lago T, Wilksch J, Zhang Y, Webb GI, Song J, Lithgow T. Comprehensive assessment and performance improvement of effector protein predictors for bacterial secretion systems III, IV and VI. Brief Bioinform 2018; 19:148-61. 59. Jana B, Fridman CM, Bosis E, Salomon D. A modular effector with a DNase domain and a marker for T6SS substrates. Nat Commun 2019; 10:3595.

60. Wang J, Yang B, Leier A, Marquez-Lago TT, Hayashida M, Rocker A, Zhang Y, Akutsu T, Chou K-C, Strugnell RA, et al. Bastion6: a bioinformatics approach for accurate prediction of type VI secreted effectors. Bioinformatics 2018; 34:2546-55.

61. Nguyen TT, Lee H-H, Park I, Seo Y-S. Genome-Wide Analysis of Type VI System Clusters and Effectors in Burkholderia Species. Plant Pathol J 2018; 34:11-22.

62. Zalguizuri A, Caetano-Anollés G, Lepek VC. Phylogenetic profiling, an untapped resource for the prediction of secreted proteins and its complementation with sequence-based classifiers in bacterial type III, IV and VI secretion systems. Brief Bioinform 2019; 20:1395402.

63. Sen R, Nayak L, De RK. PyPredT6: A python-based prediction tool for identification of Type VI effector proteins. J Bioinform Comput Biol 2019; 17:1950019.

64. Monjarás Feria J, Valvano MA. An Overview of Anti-Eukaryotic T6SS Effectors. Front Cell Infect Microbiol 2020; 10:584751.

65. Stam R, Mantelin S, McLellan H, Thilliez G. The role of effectors in nonhost resistance to filamentous plant pathogens. Front Plant Sci [Internet] 2014 [cited 2020 Oct 11]; 5. Available from: https://www.ncbi.nlm.nih.gov/pmc/articles/PMC4224059/

66. Varden FA, De la Concepcion JC, Maidment JH, Banfield MJ. Taking the stage: effectors in the spotlight. Curr Opin Plant Biol 2017; 38:25-33. 